\title{
Polycyclic aromatic hydrocarbons in wastewater, WWTPs effluents and in the recipient waters of Beijing, China
}

\author{
Weixiao Qi • Huijuan Liu • Benoît Pernet-Coudrier • \\ Jiuhui Qu
}

Received: 25 July 2012 / Accepted: 13 December 2012 /Published online: 6 January 2013

(C) Springer-Verlag Berlin Heidelberg 2013

\begin{abstract}
In this study, surface water samples from the Wenyu River and the North Canal, effluent from major wastewater treatment plants (WWTPs) in Beijing, and wastewater from open sewers that discharge directly into the river system were collected and analyzed for 16 priority USEPA polycyclic aromatic hydrocarbons (PAHs). Concentrations of these 16 PAHs ranged from 193 to $1790 \mathrm{ng} / \mathrm{L}$ in river surface waters, 245 to $404 \mathrm{ng} / \mathrm{L}$ in WWTP effluents, and 431 to $2860 \mathrm{ng} / \mathrm{L}$ in the wastewater from the small sewers. The WWTP effluent was the main contributor of dissolved PAHs to the river, while wastewater from the small sewers contributed both dissolved and suspended particulate matter-associated PAH to the river as indicated by the high dissolved organic carbon and suspended particulate matter contents in the wastewater. Although the flow from each open sewer was small, a PAH discharge as high as $44 \mathrm{~kg} /$ year could occur into the river from these types of sewers. This amount was equivalent to about $22 \%$ of the PAH loads discharged into the North Canal downstream from Beijing, whereas the remainder was mainly released by the major WWTPs in Beijing.
\end{abstract}

Keywords Polycyclic aromatic hydrocarbons - Wastewater treatment plant $\cdot$ Receiving river $\cdot$ Sewer $\cdot$ Flux $\cdot$ Sources

Responsible editor: Leif Kronberg

Electronic supplementary material The online version of this article (doi:10.1007/s11356-012-1435-6) contains supplementary material, which is available to authorized users.

W. Qi $\cdot$ H. Liu $(\bowtie) \cdot$ J. Qu

State Key Laboratory of Environmental Aquatic Chemistry,

Research Center for Eco-Environmental Sciences, Chinese

Academy of Sciences, Beijing 100085, China

e-mail: hjliu@rcees.ac.cn

B. Pernet-Coudrier

Eawag, Swiss Federal Institute of Aquatic Science

and Technology, 8600 Dübendorf, Switzerland

\section{Introduction}

Polycyclic aromatic hydrocarbons (PAHs) are ubiquitous in water bodies near urban areas due to the prolific use of fossil fuels by human civilization. The PAHs found in the rivers could come from the discharge of treated or untreated wastewater, spilled oil, atmospheric deposition, and surface runoff. PAHs found in municipal wastewater normally come from the discharge of petroleum products like petroleum fuels and lubricants, the discharge of urban storm water runoff containing PAHs from asphalt and car exhaust particles (Tanaka et al. 1990; Wang et al. 2011a, 2011b), and household wastes, which can account for 50-60\% of the total sewer load for pyrene and phenanthrene (Mattsson et al. 1991). PAHs could be discharged from effluent of wastewater treatment plants to the receiving river in dissolved phase or adsorbing to flocculant particles (Pham and Proulx 1991; Zeng and Vista 1997; Gómez et al. 2008; Qi et al. 2011). Atmospheric bulk deposition of PAHs could reach $5.2 \pm 3.89 \mu \mathrm{g} / \mathrm{m}^{2} /$ day in the BeijingTianjin region (Wang et al. 2011a).

Beijing and Tianjin are located in the region of annulus Bohai sea, which is the third largest industrial area in China. Wastewater (WW), either after treatment or without treatment, is one of the main water sources of many rivers in this area, which is now facing crises for water resources due to rapid development and 25 years of drought recorded since the 1970s. High PAH levels have been previously reported for streams in Tianjin (Shi et al. 2005; Qi et al. 2011). The occurrence and characterization of PAHs have also been reported in urban river systems in Beijing (Zhang et al. 2004; Shen et al. 2009). Fuel and biomass combustion, coke ovens, etc., are clearly the primary sources of PAHs in the rivers in the Beijing and Tianjin area (Shi et al. 2007, 2009). However, for the rivers that receive treated and untreated wastewater as their main water sources, the contribution of wastewater to PAH content has not yet been determined. Further research on occurrence 
and characterization of PAHs from wastewater discharge point sources may improve our understanding and perhaps allow quantitative evaluation of the sources of PAHs in rivers.

Prior to the 2008 Olympic Games, the Beijing authorities decided to build 10 new wastewater treatment plants (WWTPs) and laid more than $4,000 \mathrm{~km}$ of sewers to treat $90 \%$ of Beijing's wastewater. Nevertheless, to our knowledge, no monitoring program was put in place to assess how these new WWTPs improved the water quality in the river system. For this reason, determining the concentrations of anthropogenic PAHs in effluents from these WWTPs and their distribution in the rivers is also critical for evaluating this new system's capacity for reducing contaminant loading and for river management to better protect and conserve the river system.

The objectives of this study were to measure the concentrations of PAHs in effluents from the WWTPs and wastewater from small open sewers that discharge directly into rivers. This allowed us to estimate the loadings of PAHs discharged into the Wenyu River. We also collected water samples from a landscaped area to use as background samples to contrast and compare with the urban river samples.

\section{Materials and methods}

\section{Chemicals}

PAHs [naphthalene (Nap), acenaphthylene (Acy), acenaphthene (Ace), fluorene (Fl), phenanthrene (Phe), anthracene (Ant), fluoranthene (Fluo), pyrene (Pyr), benz $[a]$ anthracene (BaA), chrysene (Chry), benzo[b]fluoranthene (BbF), ben$\mathrm{zo}[k]$ fluoranthene $(\mathrm{BkF})$, benzo $[a]$ pyrene $(\mathrm{BaP})$, indeno[1,2,3$c d]$ pyrene (Ind), dibenz $[a, h]$ anthracene (DBA), benzo $[g h i]$ perylene (BghiP)] in a mixture $(200 \mu \mathrm{g} / \mathrm{mL}$ for each PAH, in dichloromethane and methanol) and perdeuterated PAHs (acenaphthene-d10, phenanthrene-d10, chrysene-d12) in a mixture $(2,000 \mu \mathrm{g} / \mathrm{mL}$ for each PAH, in dichloromethane) were purchased from AccuStandard, Inc. (New Haven, USA). Solid 2fluorobiphenyl ( $>96 \%$ ) was purchased from Aldrich Chemical Co., Inc. (Gillingham, Dorset, USA). All organic solvents used in the analysis were HPLC grade or pesticide residue grade and were used without any further purification. Neutral silica gel (0.06-0.2 mm) for chromatography was purchased from Acros Organics, Inc. (New Jersey, USA). Silica gel and alumina (100-200 mesh) were washed by ultrasonication in dichloromethane (DCM) twice for $20 \mathrm{~min}$ and then baked at 180 and $250{ }^{\circ} \mathrm{C}$ for $12 \mathrm{~h}$. Sodium sulfate was baked at $450{ }^{\circ} \mathrm{C}$ for $4 \mathrm{~h}$ and then stored in a sealed desiccator.

\section{Sample collection}

The Wenyu River originates from the Shahe reservoir (site 1) and is named the North Canal in the downstream region beyond site 5 (Fig. 1). The river converges with the Yongdingxin River at site 13, which is the Qujiadian dam located at Tianjin. The Yongdingxin River flows into Bohai Bay as shown in Fig. 1. The Wenyu River and the North Canal receives almost all of its water from the WWTPs of Beijing by way of four tributaries: the Qing River (between sites 3 and 4, Fig. 1), the Beixiao River (between sites 4 and 5), the Tonghui River (between sites 5 and 6), and the Liangshui River (between sites 6 and 7). In the upper reaches (between sites 2 and 4), untreated wastewater is discharged into the river from many small open sewers. Except the North Canal, many other rivers and canals merge into Yongdingxin River, such as Airport Drainage Canal, Beijing Drainage Canal, Chaobai New River, Ji Canal, Jinzhong River, and North Drainage Canal, etc. Yongdingxin River is one of the flooddischarging channels of Tianjin and acts as an important input path of Yongding River, North Canal, Chaobai New River, and Ji Canal into Bohai Sea.

In April and July 2009 (dry and wet seasons, respectively), samples were collected at 16 sites along the river system (Fig. 1). Samples at sites 1, 5, 9, 12, and 13 were collected monthly from April 2009 to June 2010. Background samples were collected in June 2009 from brooks in a landscaped area free from heavy traffic and were designated as U1, U2, and U3 (Table SM1). Samples from tributaries of the Wenyu River were also collected at T1 in the Tonghui River and T2 in the Liangshui River in June 2009. In addition, effluents from Beijing's five largest WWTPs (Qinghe, Jiuxianqiao, Beixiaohe, Gaobeidian, and Xiaohongmen WWTP) were collected in May 2010. Wastewater (WW) samples from small sewers discharging into the rivers were also collected in May

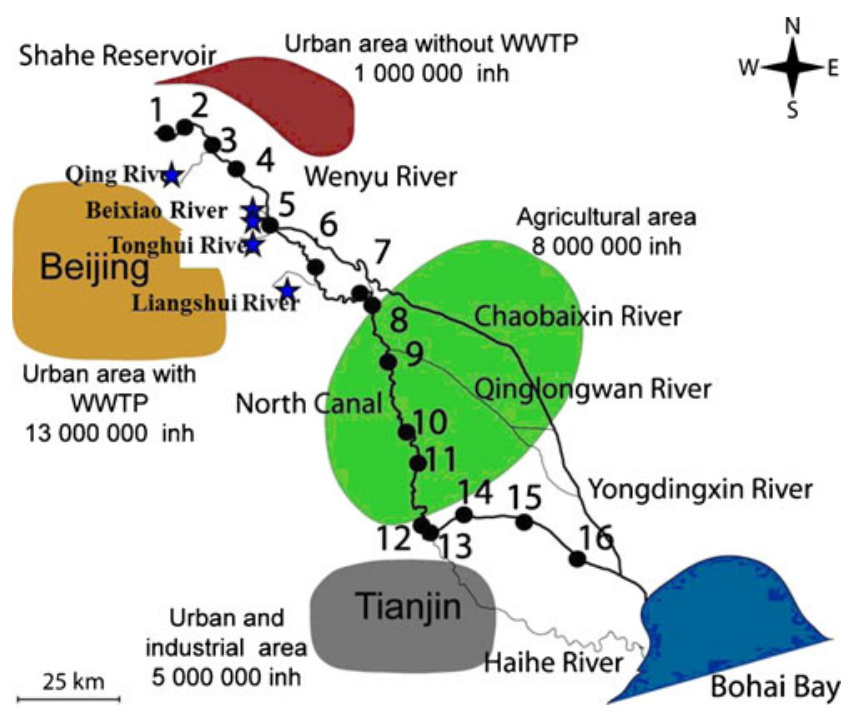

Fig. 1 Map of the locations of the sampling sites in the northern Haihe river system in Beijing and Tianjin. The star represents the location of a WWTP. From north to south, these are the Qinghe WWTP, the Beixiao WWTP, the Jiuxianqiao WWTP, the Gaobeidian WWTP, and the Xiaohongmen WWTP 
2010. The locations of these sites are presented in the Supplementary material (SM) (Table SM1).

Surface water samples were collected and stored in 4-L precleaned brown glass bottles. Back in the lab, the water samples were filtered through glass fiber filters (Millipore, $0.7-\mu \mathrm{m}$ pore size, prebaked at $450{ }^{\circ} \mathrm{C}$ for $4 \mathrm{~h}$ ). The glass fiber filters containing the suspended particulate matter (SPM) samples were stored in a refrigerator at $-18^{\circ} \mathrm{C}$ until analysis. After spiking with surrogate standards, the filtered water samples were passed through $\mathrm{C} 18$ cartridges (Supelco, USA) to extract the dissolved organic pollutants.

\section{Pretreatment procedure}

The organic pollutants retained on the $\mathrm{C} 18$ cartridges were eluted with a mixture of 1:9 hexane and DCM, followed by $5 \mathrm{~mL}$ of hexane. The SPM samples on the glass fiber filters were freeze-dried, weighed, and then spiked with surrogate standards, followed by accelerated solvent extraction (ASE300, Dionex, USA) in a mixture of 1:1 DCM and acetone for $10 \mathrm{~min}$ for two static cycles at $120{ }^{\circ} \mathrm{C}$ and 1,500 psi.

The eluate or extract of each sample was concentrated, solvent exchanged, and purified on 1:2 alumina/silica gel as previously reported by Mai et al. (2002). The internal standard (2-fluorobiphenyl) was added to the samples prior to instrumental analysis.

\section{Instrumental analysis}

The concentrations of the 16 priority PAHs were determined by GCMS using an Agilent 7890A GC equipped with a 5975C mass selective detector under the selected ion monitoring mode. A HP-5 silica fused capillary column (30 m× $0.25 \mathrm{~mm}$ inner diameter $\times 0.25 \mu \mathrm{m}$ film thickness) was used, with helium as the carrier gas. The operation parameters were the same as those reported by (Qiao et al. 2006).

Dissolved organic carbon (DOC) contents of water samples were analyzed with a TOC- $\mathrm{V}_{\mathrm{CPH}}$ total organic carbon analyzer (Shimadzu, Japan). The limit of detection for water samples was $0.5 \mathrm{mg} / \mathrm{L}$.

\section{Quality assurance and quality control}

The quality assurance (QA) and quality control (QC) procedures included analysis of method blanks, matrix duplicated spikes, and sample duplicates. The six-point calibration line was determined and calibrated daily. The recoveries of acenaphthene-d10, phenanthrene-d10, and chrysene-d12 for water samples were $75 \pm 18,91 \pm 29$, and $63 \pm 9 \%$, respectively. For SPM samples, these recoveries were $99 \pm 21,91 \pm 11$, and $90 \pm 7 \%$, respectively. The concentrations were not corrected by the surrogate recovery data. The method detection limit and RSD for water samples were 9-25 ng/L and 0.4-10.0\%, respectively, and for solid samples were $2-14 \mathrm{ng} / \mathrm{g}$ and $1.1-6.8 \%$, respectively.

Load calculation

Monthly PAH load in the river was calculated with monthly water discharge averaged from daily water discharge multiplied by concentration of PAHs for the month. The annual load of PAHs was calculated by summing monthly PAH load in the whole sampling year. The atmospheric deposition flux to the river was estimated roughly using the deposition flux in Beijing-Tianjin reported by Wang et al. (2011a) multiplied by the surface area of the river.

\section{Results and discussion}

PAH occurrence in the river, sewers, and WWTPs

Box plots in Fig. 2 show the maximum, median, minimum, $25 \%, 75 \%$, and mean concentrations of the PAHs in the influent (INF)/effluent (EFF) samples from WWTPs, WW samples from open sewers, and samples from the receiving river (R). Of PAHs in INF samples 75-90\% could be removed in the five WWTPs investigated in this study (Fig. 2, a and b). The effluent PAH levels in the present study (245-404 ng/L) were similar to those reported in other studies: $120-630 \mathrm{ng} / \mathrm{L}$ in WWTPs in Korea (Lee et al. 2011), $1,120 \mathrm{ng} / \mathrm{L}$ in a WWTP in Italy (Busetti et al. 2006), and $418 \pm 181 \mathrm{ng} / \mathrm{L}$ in a WWTP in Canada (Pham and Proulx 1991). Dissolved PAHs in the WW samples (550-2,020 ng/L) had higher concentrations than those in EFF (185-400 ng/L) and river samples (100-800 ng/L), but similar level with those in INF samples $(800-1,610 \mathrm{ng} / \mathrm{L})$ from WWTPs (Fig. 2, a). For the SPM samples, the PAH concentrations in the river, INF and EFF of WWTPs, and WW samples were between 1.6-24.0, 4-39.1, 3.8-41.5, and $3.1-7.4 \mu \mathrm{g} / \mathrm{g}$, respectively (Fig. 2, c). This was highly related with the suspended matter contents in the water samples which would be discussed further later. Totally, PAHs in the receiving rivers seemed to be mainly influenced by the discharging of WWTPs which diluted the wastewater from the small open sewers.

As untreated wastewater, both the INF and WW samples, which had higher suspended particulate matters, showed lower dissolved PAH ratios (around 59 and $63 \%$ ) based on total PAHs, when compared with EFF samples (around $80 \%$, Fig. SM1). The percentage of dissolved PAHs versus total PAHs in samples from the Shahe reservoir $(70 \pm 5 \%$, R1 in Fig. SM1) was higher than that in other river samples (Fig. SM1). This can be easily explained since some of the PAH-containing particles may settle in the reservoir. At site 5 

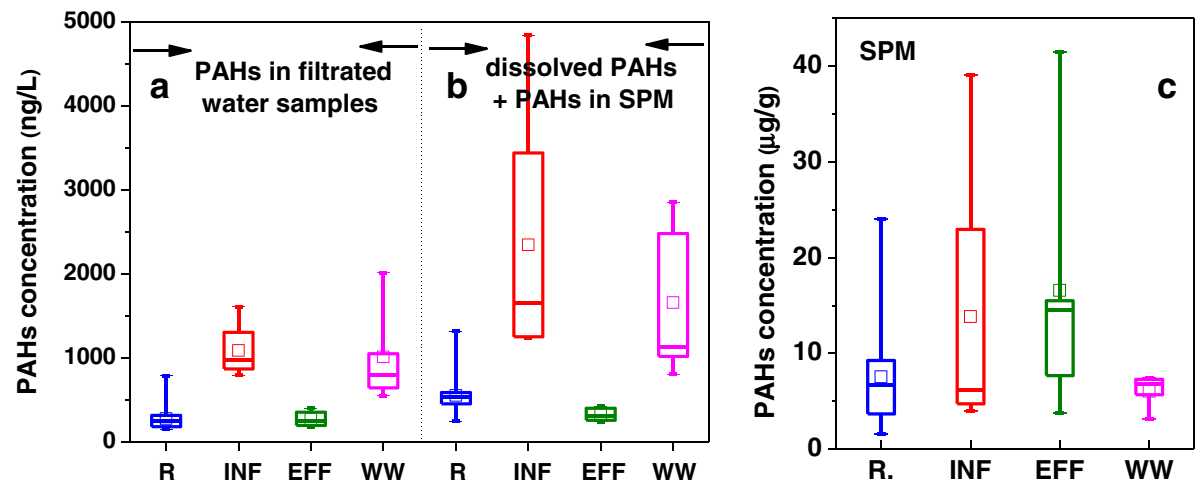

Fig. 2 Concentrations of 16 PAHs in filtered water samples (a), SPM $(c)$, the sum of both $(b)$ from $R$ (river samples), INF (influent from wastewater treatment plants), $E F F$ (effluent from wastewater treatment plants), $W W$ (wastewater from small sewers). Boxes show the range

(R5 in Fig. SM1) where WWTP discharge contributed $80 \%$ of the water flow in the river (Pernet-Coudrier et al. 2012), the percentage of the dissolved PAH $(60 \pm 11 \%)$ decreased as a result of mixing of WWTP effluent and untreated WW. The percentage of dissolved PAHs at site 9 (R9 in Fig. SM1), which was located in a rural area further downstream from Beijing, was low ( $47 \pm 5 \%$, Fig. SM1) when compared with the other river samples, and this phenomenon could not be explained only by the mixing of WWTP and WW. Clearly, another source of PAHs and/or more probably a process like adsorption by particles was occurring at this site.

In order to investigate the factors influencing the form of PAHs occurring in the wastewater and receiving rivers, the relation between PAH content with DOC and SPM contents was analyzed. The contents of PAHs in the river samples showed a weak correlation $(r=0.34)$ with DOC concentrations (Fig. 3, left). Total PAH concentrations in SPM, if the aqueous volumes were used, were highly correlated $(r=0.74, p<0.001)$ with SPM contents in surface water (Fig. 3 , right). The WW showed higher contents of SPM and SPM-associated PAHs than most river samples, while WWTP effluents showed lower levels. Based on this analysis, WW appeared to contribute both dissolved and SPM-associated PAHs to the river (Fig. 2), while WWTPs mainly contributed dissolved PAHs to the river. Additionally, SPM content in the EFF from WWTPs was between the 25th and 75th percentiles. The whiskers extend from the edge of the box to the minimum and maximum data values. The horizontal line and the square inside indicate the median value and the mean value, respectively

important for controlling the PAH discharge into the receiving waters, especially for the particulate-associated PAHs.

PAH characterization in the rivers in comparison with background sites

The PAH concentrations varied between the background sites and the urban river sampling sites. Some individual PAHs in the background SPM samples showed high concentrations per mass unit of SPM. Concentrations of Nap, Fl, Phe, Ant, Fluo, Chry, and BbF at U1 were 14.3, 3.3, 6.3, $0.9,1.4,1.3$, and $1.7 \mu \mathrm{g} / \mathrm{g}$, respectively (Fig. 4a). In comparison, concentrations of Nap, Fl, Phe, and Ant at T2 were $0.9,0.4,0.9$, and $0.2 \mu \mathrm{g} / \mathrm{g}$, respectively (Fig. 4a). However, the SPM contents at the background sites, U1 and U3, were much lower, 2.18 and $4.28 \mathrm{mg} / \mathrm{L}$, respectively, when compared to sites $1,5, \mathrm{~T} 1,6$, and $\mathrm{T} 2$ along the river, which were $11.53,29.91,35.53,17.29$, and $28.05 \mathrm{mg} / \mathrm{L}$, respectively.

Generally, samples in the background had low DOC ( $2.3 \mathrm{mg} / \mathrm{L}$ at U1) and low SPM content, but high PAH content per mass unit of SPM, especially for Nap, Fl, Phe, Ant, Fluo, Chry, and BbF. PAHs in the urban rivers had more complex sources compared to the brooks in the landscaped area and had a higher content of individual PAHs per volume unit of water.
Fig. 3 Correlation of total PAH concentrations with DOC contents in the water samples from the rivers, WW from small sewers, and effluent samples from WWTPs $(l e f t)$; correlation of total PAH concentrations in SPM samples with SPM contents in surface water ( $p$ level of significance; $r$ Pearson correlation coefficient)
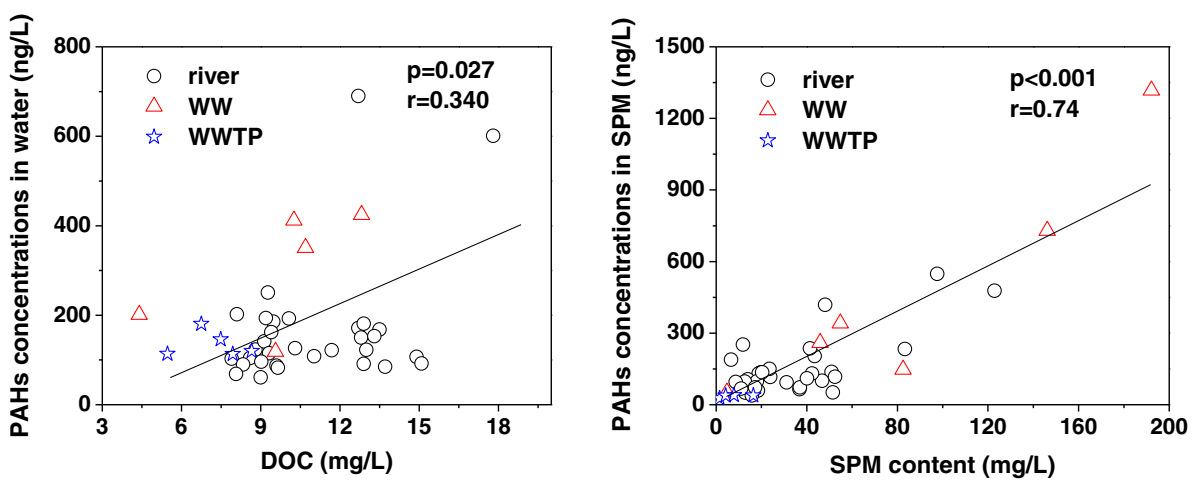
Fig. 4 Levels of PAHs in SPM from the background, upstream regions of Shahe reservoir, the Wenyu River, the North Canal, and Wenyu River tributaries (June 2009)
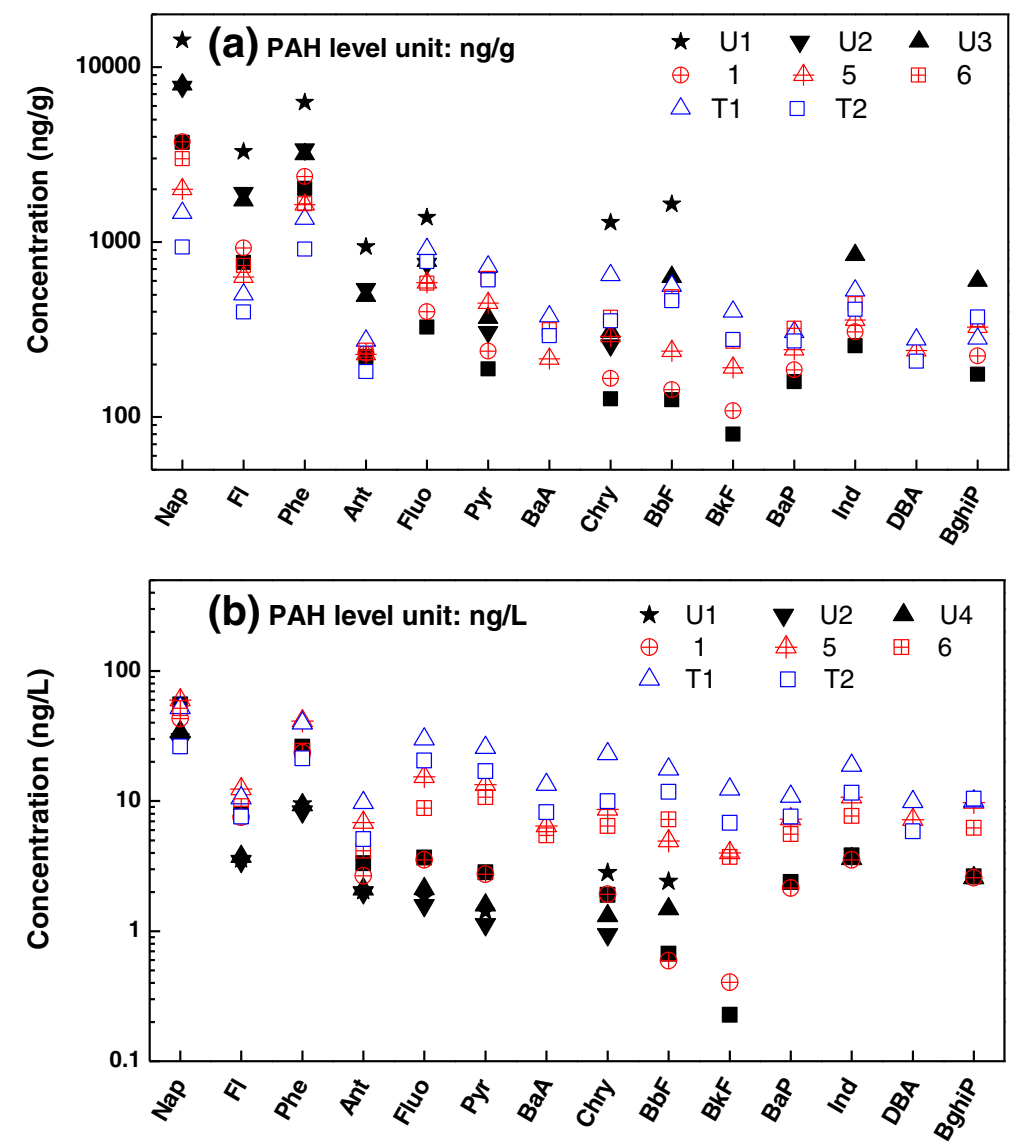

The Ant/178 against Fluo/202 ratios in the SPM samples are plotted in Fig. 5, in order to further discuss different PAH origins in urban rivers and landscaped brooks. The PAHs in the brooks in the landscaped area were most likely derived from coal and wood combustion, as the ratios of Ant/178 and Fluo/202 were higher than 0.1 and 0.5, respectively. The PAHs in open sewers probably originated from both pyrogenic and petrogenic sources. Ratios of Ant/178 and Fluo/202 in most of the river samples were higher than 0.1 and 0.5 , respectively, suggesting a predominance of

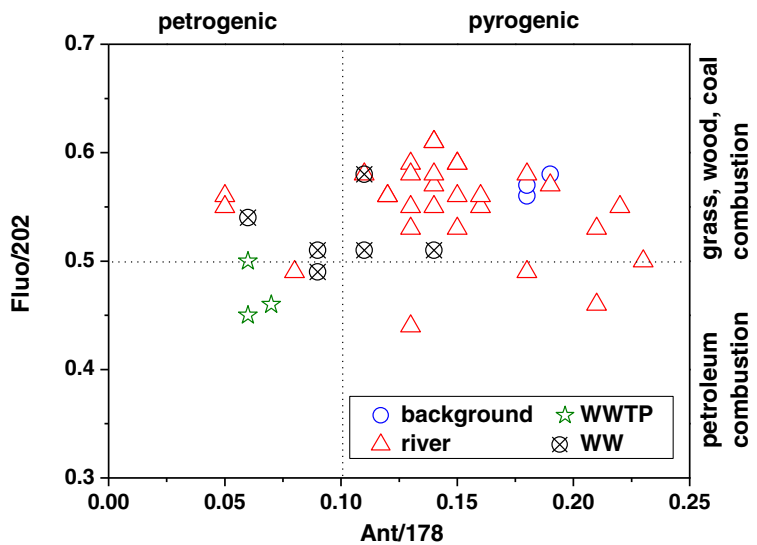

Fig. 5 PAH cross plots for the ratios of Ant/178 versus Fluo/202 in SPM from background, river, WWTP effluent, and WW samples pyrogenic source for these PAHs. For PAHs in WWTP effluent samples, which have undergone treatment processes in the plants, the values for ratios of isomers were lower than those seen for the other samples (Fig. 5). Based on the Ant/ 178 and Fluo/202 ratios, the effluents from WWTPs were not the only major PAH source of the rivers. The small open sewers probably contribute substantial pyrogenic PAHs into the rivers. However, the amounts of particulate PAHs were not solely explained by the input from WW, and the types of PAHs found in the river indicated a greater pyrogenic contribution than that supplied by the WW. Therefore, other sources of PAH should be considered, such as atmospheric deposition and surface runoff, as these may influence the signature of PAHs, especially in this river with its very low flow regime, i.e., it is a very large and shallow river.

Spatial distribution of PAHs in the rivers

As Nap constituted a high percentage of the total PAHs, it was excluded in the following analysis of spatial distribution variations. The PAH concentrations in filtrates and SPM from the Wenyu River showed lower concentrations at the dam sites (Fig. SM2), such as site 1 (the Shahe Dam on the Wenyu River), site 7 (the Yangwa Dam on the North Canal), and site 13 (the Qujiadian Dam on the Yongdingxin River), which were often closed. This was particularly marked for 
SPM-associated PAHs when expressed in units of microgram per gram (Fig. SM2 (b)). This phenomenon could be explained by hydrology, as most SPM would settle if the dams were closed most of the time. The SPM-associated PAH peak seen at site 3 could be explained by discharge from wastewater, as many small sewers (e.g., sites WW1, WW2, and WW3) were located between sites 2 and 3 on the river. In contrast, the decrease seen at site 4 could be explained by dilution from the Qinghe WWTP, which discharged between sites 3 and 4 .

\section{Assessment of PAH inputs from WWTPs}

PAH flux ranges at sites $1,5,9$, and 13 were $0.5-3.2,2.3-$ $27.1,3.5-34.5$, and $0.4-9.0 \mathrm{~kg} / \mathrm{month}$, respectively. The column in Fig. SM3 shows the average results for the PAH fluxes at these five sites.

The loading of PAHs discharged from the Beijing WWTPs was calculated from PAH concentrations measured in the present study, multiplied with the daily flow from the WWTPs. The PAH flux at each studied WWTP was between 1.5 and $7.3 \mathrm{~kg} / \mathrm{month}$ for all 16 PAHs. This level was the same magnitude as the PAH flux in the rivers in the dry seasons, which was between 0.4 and $5.5 \mathrm{~kg} /$ month.

As most of the WWTPs shown in Fig. SM3 do not discharge directly into the Wenyu River, but discharge into the stretch streams, assessing the exact PAH loading contribution of the WWTPs to the Wenyu River was difficult to assess quantitatively. Estimation of the PAH mass emission from countless small sewers was also difficult, as their water discharges are small and irregular. However, PernetCoudrier et al. (2012) evaluated the total flow of these sewage discharges to be $1.3 \mathrm{~m}^{3} / \mathrm{s}$. If a mean flow of $1.3 \mathrm{~m}^{3} / \mathrm{s}$ and the median PAH concentration of $1,080 \mathrm{ng} / \mathrm{L}$ are assumed from the data from this study, the average mass emission of PAHs from the small sewers was roughly $3.6 \mathrm{~kg} / \mathrm{month}$ or $44 \mathrm{~kg} /$ year. From a watershed-scale perspective, the fluxes from untreated WW (44 kg/year) are diluted by the discharge of the WWTPs (210 kg/year), but they still contribute about $22 \%$ of the loads measured at site 9 (190 kg/year) downstream from the Beijing megacity. Nevertheless, the loads might have been underestimated due to sedimentation in the river, especially due to the very low flow regime of this river. The atmospheric deposition flux of PAHs to the river was estimated roughly using the deposition flux data in the Beijing-Tianjin area reported by Wang et al. (2011a). In the river from site 1 to site 9, which is $90 \mathrm{~km}$ and about $50 \mathrm{~m}$ wide, the PAH atmospheric deposition was about $14 \mathrm{~kg}$ /year. If not considering the sedimentation of PAHs in the river, PAHs from atmospheric deposition contributed about $7 \%$ of the loads at site 9 . Although the PAH contribution from atmospheric deposition was not as great as wastewater discharge, the high Ant/178 ratio in atmospheric deposition samples (Wang et al. 2011a) proved its pyrogenic PAH contribution to the rivers.

\section{Conclusion}

Concentrations of 16 PAHs ranged from 193 to $1,790 \mathrm{ng} / \mathrm{L}$ in Wenyu River and the North Canal, 245 to $404 \mathrm{ng} / \mathrm{L}$ in WWTP effluents, and 431 to $2,860 \mathrm{ng} / \mathrm{L}$ in the wastewater from the small sewers. The WWTP effluent was the main contributor of dissolved PAHs to the river, while wastewater from the small sewers contributed both dissolved and suspended particulate matter-associated PAH to the river. SPM content in the WWTP effluent was important for controlling the PAH discharge into the receiving waters. Although the flow from each open sewer was small, PAH discharge from many of these types of sewers was not negligible. In Beijing, the fluxes from untreated WWs (44 kg/year) were diluted by the discharge of the WWTPs (210 kg/year) and contributed about $20 \%$ of the loads in the river.

Acknowledgments This work was supported by the Key Program of National Natural Science Foundation of China (51138006), the SinoSwiss Science and Technology Cooperation for Joint Research Projects (GJHZ0908), and the State Key Joint Laboratory of Environment Simulation and Pollution Control (12L02ESPC). The authors thank Senior Scientist Michael Berg at Eawag, Switzerland, for his help in language improvement of this paper.

\section{References}

Busetti F, Heitz A, Cuomo M, Badoer S, Traverso P (2006) Determination of sixteen polycyclic aromatic hydrocarbons in aqueous and solid samples from an Italian wastewater treatment plant. J Chromatogra A 1102:104-115

Gómez A, Rubio S, Bendito D (2008) Determination of priority carcinogenic polycyclic aromatic hydrocarbons in wastewater and surface water by coacervative extraction and liquid chromatographyfluorimetry. J Chromatogra A 1203:168-176

Lee IS, Sim WJ, Kim CW, Chang YS, Oh JE (2011) Characteristic occurrence patterns of micropollutants and their removal efficiencies in industrial wastewater treatment plants. J Environ Monitor 13:391-397

Mai BX, Fu JM, Sheng GY, Kang YH, Lin Z, Zhang G, Min YS, Zeng EY (2002) Chlorinated and polycyclic aromatic hydrocarbons in riverine and estuarine sediments from Pearl River Delta, China. Environ Pollut 117:457-474

Mattsson J, Avergård I, Robinson P (1991) Priority pollutants, heavy metals and main constituents in the domestic sewage from residential areas in Gothenburg. Vatten 47:204-211

Pernet-Coudrier B, Qi WX, Liu HJ, Müller B, Berg M (2012) Sources and pathways of nutrients in the simi-aird region of Beijing-Tianjin region, China. Environ Sci Technol. doi:10.1021/es3004415

Pham TT, Proulx S (1991) PCBs and PAHs in the Montreal urban community (Quebec, Canada) wastewater treatment plant and 
in the effluent plume in the St Lawrence river. Water Res 31:1887-1896

Qiao M, Wang CX, Huang SB, Wang DH, Wang ZJ (2006) Composition, sources, and potential toxicological significance of PAHs in the surface sediments of the Meiliang Bay, Taihu Lake, China. Environ Int 32:28-33

Qi WX, Liu HJ, Qu JH, Hu CZ, Lan HC, Berg M, Ren HM, Xu W (2011) Polycyclic aromatic hydrocarbons in effuents from wastewater treatment plants and receiving streams in Tianjin, China. Environ Monit Assess 177:467-480

Shen Q, Wang YK, Zhang W, Zhang CS, Wang JX (2009) Characterization and sources of PAHs in an urban river system in Beijing, China. Environ Geochem Hlth 31:453-462

Shi Z, Tao S, Pan B, Fan W, He XC, Zuo Q, Wu SP, Li BG, Cao J, Liu WX, Xu FL, Wang XJ, Shen WR, Wong PK (2005) Contamination of rivers in Tianjin, China by polycyclic aromatic hydrocarbons. Environ Pollut 134:97-111

Shi Z, Tao S, Pan B, Liu WX, Shen WR (2007) Partitioning and source diagnostics of polycyclic aromatic hydrocarbons in rivers in Tianjin, China. Environ Pollut 146:492-500
Tanaka H, Onda T, Ogura N (1990) Determination of polycyclic aromatic hydrocarbons in urban street dusts and their source materials by capillary gas chromatography. Environ Sci Technol $24: 1179-1186$

Wang WT, Simonich S, Giri B et al (2011a) Spatial distribution and seasonal variation of atmospheric bulk deposition of polycyclic aromatic hydrocarbons in Beijing-Tianjin region, North China. Environ Pollut 159:287-293

Wang WT, Simonich S, Giri B et al (2011b) Atmospheric concentrations and air-soil gas exchange of polycyclic aromatic hydrocarbons (PAHs) in remote, rural village and urban areas of Beijing-Tianjin region, North China. Sci Total Environ 409:2942-2950

Zeng EY, Vista LC (1997) Organic pollutants in the coastal environment off San Diego, California. 1. Source identification and assessment by compositional indices of polycyclic aromatic hydrocarbons. Environ Toxicol Chem 16:179-188

Zhang ZL, Huang J, Yu G, Hong HS (2004) Occurrence of PAHs, PCBs and organochlorine pesticides in the Tonghui River of Beijing, China. Environ Pollut 130:249-261 